\title{
Optimal strategies for driving a mobile agent in a "guidance by repulsion" model
}

\author{
R. Escobedo ${ }^{1,2,3,}$, A. Ibañez ${ }^{2}$, E.Zuazua ${ }^{4}$ \\ ${ }^{1}$ CRCA - Centre de Recherches sur la Cognition Animale, \\ Université Paul Sabatier, 118 Route de Narbonne, 31062 Toulouse, France. \\ ${ }^{2}$ BCAM - Basque Center for Applied Mathematics, Alda. Mazarredo 14, 48009 Bilbao, \\ Spain. \\ ${ }^{3}$ AEPA-Euskadi, Pza. Celestino María del Arenal 14, 48015 Bilbao, Spain. \\ ${ }^{4}$ Departamento de Matemáticas, Universidad Autónoma de Madrid, Cantoblanco, 28049 \\ Madrid, Spain. \\ *corresponding author: escobedor@gmail.com
}

\begin{abstract}
We present a guidance by repulsion model based on a driver-evader interaction where the driver, assumed to be faster than the evader, follows the evader but cannot be arbitrarily close to it, and the evader tries to move away from the driver beyond a short distance. The key ingredient allowing the driver to guide the evader is that the driver is able to display a circumvention maneuver around the evader, in such a way that the trajectory of the evader is modified in the direction of the repulsion that the driver exerts on the evader. The evader can thus be driven towards any given target or along a sufficiently smooth path by controlling a single discrete parameter acting on driver's behavior. The control parameter serves both to activate/deactivate the circumvention mode and to select the clockwise/counterclockwise direction of the circumvention maneuver. Assuming that the circumvention mode is more expensive than the pursuit mode, and that the activation of the circumvention mode has a high cost, we formulate an optimal control problem for the optimal strategy to drive the evader to a given target. By means of numerical shooting methods, we find the optimal open-loop control which reduces the number of activations of the circumvention mode to one and which minimizes the time spent in the active mode. Our numerical simulations show that the system is highly sensitive to small variations of the control function, and that the cost function has a nonlinear regime which contributes to the complexity of the behavior of the system, so that a general openloop control would not be of practical interest. We then propose a feedback control law that corrects from deviations while preventing from an excesive use of the circumvention mode, finding numerically that the feedback law significantly reduces the cost obtained with the open-loop control.
\end{abstract}

Keywords: Guidance by repulsion, Driver-evader agents, Optimal strategies, Feedback control law, Nonlinear dynamics, Numerical simulations 


\section{Introduction}

"Follow me" (FM) is probably the most natural strategy to solve the guidance problem, that is, to guide something or somebody along a given trajectory or towards a specific target, whether physically along geographical paths (streets, roads, buildings), conceptually (language learning, politics) or even spiritually (religion, social networks). The success of the FM strategy is based on the effect of the attraction that the guide (the leader, the driver) exerts on the guided (followers, driven). Also successfull is the "Do as I do" strategy, based on imitation (of behavior) or alignment (of opinion), and used in the above mentioned fields, among others (social learning).

Less expected is the effectiveness of the opposite strategy, namely, "move away from $m e "$ (MA), based on repulsive interactions. That repulsion can serve to guide something or somebody is shown by nature itself, not only by means of gradient fields (electromagnetic, temperature or chemical fields), where attraction towards high densities can be viewed as repulsion from low densities, but also by specifically repelling targets or agents. In neural development, axonogenesis takes place by combining attractive and repulsive guidance, so that the axon growth follows guidance cues presented by chemoattractant and chemorepellent molecules located in the environment of the cell [1, 2]. In animal herding, sheepdogs are used to guide sheep flocks through a repulsive force that dogs exert on sheep [3]. Guidance cues can also be magnetic, as in drug targeting [4, 5], thermotactic, as in sperm guidance [6], cognitive, as in crowd motion and traffic flow [7, 8] or in opinion formation [9], acoustic signals, as in animal alarm calls or instrumental conditioning [3], food trail pheromones in ants [10] (chemical at a scale larger than the cell), etc.

Attractive and alignment guidance problems are being studied for a long time by means of agent based models [11, 12, 13, 14, 15, 16, 17, 18, 19, 20, 21, 22, 23, 24, 25, 26, 27, with attention to guidance by leadership [28, 29, 30], and optimal strategies to minimize time guidance (optimal evacuation times) or distance travelled by the agents have been found for several systems [32, 31, 34, 35, 36].

Very recently, in 2015, the optimal strategy for a flocking model to reach a target point or to follow a given trajectory through attractive and alignment guidance has been presented [37]. In this model, individuals from the flock interact through attractiverepulsive and alignment forces with the rest of individuals. Interactions are symmetric, except for one specific individual -the leader- which exerts on each other individual an extra attractive force. The result is that the leader is followed by the flock, so that, by controlling the behavior of the leader, a FM strategy can be used to make the flock reach a given target or move along a given trajectory.

Repulsive forces in attractive-repulsive models have mostly been considered for collision avoidance with obstacles or with other agents. Guidance by repulsion has received much less attention, which is reduced, to our knowledge, to the above mentioned axonogenesis and animal herding. Also very recently, in 2015, an agent based model has been introduced to describe a so-called defender-intruder interaction, where repulsion is used by a defender to expel an intruder away as far as possible from a protected target [38]. The authors in [38] find an optimal MA strategy, which not always consists in approaching 
the intruder as close as possible, but to simply drive the intruder away beyond a short distance of security. Repulsion in the intruder-defender interaction is qualitatively different than in collision avoidance or in interception [39, 40], where the attractive and/or alignment forces determine (most of) the behavior.

Defender-intruder problems fall into the category of "conflicting interactions" [38], which are well described by the classical pursuit-evasion (PE) framework [41, 42. The simplest scenario for a PE interaction consists of a single pursuer that follows and tries to capture a single evader that tries to escape to infinite from the pursuer [38].

Although our interest does not focus on conflicting interactions, we adopt here the PE framework. The guidance by repulsion can indeed be described with the simple twoagents PE framework, provided two considerations are taken into account: first, the guide is not exactly a pursuer, as it often separates from the direction towards the evader and the guide cannot be arbitrarily close to the evader, and second, the agent to be guided is not exactly an evader, as it doesn't try necessarily to escape to infinite but simply moves away a short distance from the repelling guide.

We present here a guidance by repulsion model based on the two-agents PE framework. We will refer to the guiding agent as the driver, which tries to drive the evader. The driver thus follows the evader but cannot be arbitrarily close to it. This is especially interesting if the driver cannot approach the evader or contact between agents should be avoided (because of chemical reactions, animal conflict, etc). The evader moves away from the driver but doesn't try to escape beyond a not so large distance. The driver is of course faster than the evader. At a critical short distance, the driver can display a circumvention maneuver around the evader that forces the evader to change the direction of its motion. Thus, by adjusting the onset and offset of the circumvention maneuver, the evader can be driven towards a desired target or along a given trajectory. Our goal is to find optimal strategies to drive the evader in the most efficient way.

We use an inertial model where interactions between agents take place through asymmetric newtonian forces. The asymmetry consists in that one agent is attracted and repulsed, while the other is simply repulsed. This kind of interaction has been coined "anti-newtonian" by Sprott [19] and others [20, 38]. Thus, velocities are not constant (they depend on the state of the system), and no alignment forces are considered.

We denote by $D$ and $E$ (and indexes $d$ and $e$ ) the driver and the evader agents respectively. Both agents obeys the Newton's second law, that is, $\dot{\vec{u}}_{i}(t)=\vec{v}_{i}(t)$ and $m_{i} \dot{\vec{v}}_{i}(t)=\overrightarrow{\mathcal{F}}_{i}(t)$, where $\vec{u}_{i}(t)=\left(u_{i}^{x}(t), u_{i}^{y}(t)\right) \in \mathbb{R}^{2}, \vec{v}_{i}(t)=\left(v_{i}^{x}(t), v_{i}^{y}(t)\right)=\in \mathbb{R}^{2}, m_{i} \in \mathbb{R}^{+}$ and $\overrightarrow{\mathcal{F}}_{i}(t)=\left(\mathcal{F}_{i}^{x}(t), \mathcal{F}_{i}^{y}(t)\right) \in \mathbb{R}^{2}$ denote the position vector, the velocity vector and the mass of agent $i$, and the resultant force to which agent $i$ is subject, respectively, for $i=d, e$.

The force acting on the evader $\overrightarrow{\mathcal{F}}_{e}(t)$ has only one component, which is in the direction of escape from the pursuer, $\vec{u}_{d}-\vec{u}_{e}$. The force acting on the pursuer $\overrightarrow{\mathcal{F}}_{d}(t)$ has a component collinear to $\overrightarrow{\mathcal{F}}_{e}(t)$ and a lateral perpendicular component $\left(\vec{u}_{d}-\vec{u}_{e}\right)^{\perp}$ which allows the pursuer to surround the evader, therefore forcing the evader to change the direction of escape. Here $(x, y)^{\perp}=(-y, x)$. 
The perpendicular component of the force acting on the pursuer $\overrightarrow{\mathcal{F}}_{d}(t)$ can be switched on and off by means of the control parameter $\kappa(t)$ which takes values on $\{-1,0,1\}$. The control parameter $\kappa(t)$ is the key ingredient of the model, as it determines the behavior of the pursuer, which in turn determines the behavior of the evader. The resulting dynamical system can be considered as a driver-evader system with two operating modes controlled by a single parameter.

Contents of the paper and sketch of the results Section titles are self-explanatory. In Sec. 2, we introduce the model equations and parameters, describing in detail the interactions between agents and with the environment. We show that the driver-evader system can be viewed as having two operating modes controlled by a single parameter, so that the system can be moved from one state to another in order to make the driver to guide the evader from any point to any other point. We study the controllability of the system, and prove that, although the system is not fully controllable, agents positions remain asymptotically close to each other (i.e., an agent can not go to infinity).

In Sec. 3, we consider what are the optimal strategies which allow the driver to guide the evader to a desired target. Activating a system has a cost, as well as keeping it in the active mode. As an illustrating example, the driver can be viewed as a spacecraft with two lateral propellers whose ignition process and fuel consumption are very high with respect to the consumption of the back propeller. Our interest is in reducing the cost by minimizing 1) the number of activation and 2) the time of use of the lateral propellers.

To do that, we formulate an optimal control problem $(O C P)$ for a cost functional including these two costs. Whe then find, in Sec. 3.1, the (unique) optimal strategy allowing the reach the target in an initially active system $\left(\kappa\left(t_{0}\right)=1\right)$, and the (unique) optimal strategy which reduces the length of the time interval $\left[t_{\mathrm{ON}}, t_{\mathrm{OFF}}\right]$ during which the control parameter has a non-zero value. This second strategy also reduces the number of manipulations of the control to two (one switch on and one switch off). In this minimization process, the cost of back propellers (which are always switched on, the driver is a self-propelled agent which is always attracted by the evader) is neglected compared to the cost of using the lateral propellers, so that there is not (too much) concern by long trajectories or long execution times where lateral propellers are switched off.

These open-loop controls are subject to the reproducibility of the initial and environmental conditions and to uncertainty about the model [32, 31]. In fact, we show here in detail that the system is highly sensitive to small variations of the values of the control, thus suggesting the appropriateness of the use of closed-loop controls that can afford for the random perturbations arising in real systems.

We then present in Sec. 3.2 a feedback control law allowing to drive the evader to any desired target with an arbitrary accuracy. This feedback law is especially advantageous as it yields a similar cost than the open-loop controls, in real conditions (i.e., under perturbations). Moreover, the feedback control law provides an excellent insight for the search of a control function yielding a substantially lower cost; as an example, we report numerical simulations of a case in which the resulting cost is reduced almost a $60 \%$ of the cost provided by the open-loop controls. 
Finally, in Sec. 4, we present our conclusions and we comment on future directions. in the last section

\section{Model formulation and first analysis}

\section{$2.1 \quad$ Equations and parameters}

The system consists of 8 ordinary differential equations (ODEs) and 8 initial conditions for the components $u_{i}^{x}(t), u_{i}^{y}(t), v_{i}^{x}(t)$ and $v_{i}^{y}(t)$, for $i=d$, e. Expanding the resultant force acting on the $i$ th-agent $\overrightarrow{\mathcal{F}}_{i}\left(\vec{u}_{d}(t), \vec{u}_{e}(t), \vec{v}_{d}(t), \vec{v}_{e}(t)\right)$, the system can be written as the following 4 vectorial ODEs with 4 initial conditions:

$$
\begin{aligned}
\dot{\vec{u}}_{d}(t)= & \vec{v}_{d}(t) \\
\dot{\vec{u}}_{e}(t)= & \vec{v}_{e}(t) \\
\dot{\vec{v}}_{d}(t)=\frac{1}{m_{d}} & {\left[-C_{D}^{E} \frac{\vec{u}_{d}(t)-\vec{u}_{e}(t)}{\left\|\vec{u}_{d}(t)-\vec{u}_{e}(t)\right\|^{2}}\left(1-\frac{\delta_{c}^{2}}{\left\|\vec{u}_{d}(t)-\vec{u}_{e}(t)\right\|^{2}}\right)\right.} \\
& \left.\quad-C_{\mathrm{R}} \frac{\delta_{1}^{4}}{\left\|\vec{u}_{d}(t)-\vec{u}_{e}(t)\right\|^{4}}\left(\vec{u}_{d}(t)-\vec{u}_{e}(t)-\kappa(t) \delta_{2} \frac{\left(\vec{u}_{d}-\vec{u}_{e}\right)^{\perp}}{\left\|\vec{u}_{e}(t)-\vec{u}_{d}(t)\right\|}\right)-\nu_{d} \vec{v}_{d}(t)\right] \\
\dot{\vec{v}}_{e}(t)= & \frac{1}{m_{e}}\left[C_{E}^{D} \frac{\vec{u}_{e}(t)-\vec{u}_{d}(t)}{\left\|\vec{u}_{e}(t)-\vec{u}_{d}(t)\right\|^{2}}-\nu_{e} \vec{v}_{e}(t)\right] \\
\vec{u}_{d}\left(t_{0}\right)= & \vec{u}_{d}^{0}, \quad \vec{u}_{e}\left(t_{0}\right)=\vec{u}_{e}^{0}, \quad \vec{v}_{d}\left(t_{0}\right)=0 \quad \text { and } \quad \vec{v}_{e}\left(t_{0}\right)=0
\end{aligned}
$$

Interactions between agents are as follows.

The expression between brackets in Eq. (3) consists of two terms with respective coefficients $C_{D}^{E}$ and $C_{R}$. The first term corresponds to the long-range attraction and short-range repulsion force that the evader exerts on the driver. Here $\delta_{c}$ is the distance at which the attraction balances the repulsion: if $\left\|\vec{u}_{e}(t)-\vec{u}_{d}(t)\right\|>\delta_{c}$, then the evader attracts the driver (and therefore the driver accelerates towards the evader), while if $\left\|\vec{u}_{e}(t)-\vec{u}_{d}(t)\right\|<\delta_{c}$, then the driver is repulsed by the evader (and the driver decelerates in the direction opposed to the evader).

The term with coefficient $C_{R}$ corresponds to the circumvention force, which as a component collinear to the attraction-repulsion interaction with the evader, given by $\vec{u}_{d}-\vec{u}_{e}$, and a component perpendicular to this direction, denoted by $\left(\vec{u}_{d}-\vec{u}_{e}\right)^{\perp}$, where $(x, y)^{\perp}=(-y, x)$. The circumvention force is thus a force that attracts the driver towards one of the two sides of the evader, where the side is determined by the sign of the parameter $\kappa(t)$.

The model considers two other critical distances, $\delta_{1}$, which is the (short) distance at which the intensity of the circumvention force is effective, and $\delta_{2}$, which denotes the distance between agents left by the driver during the circumvention maneuver. In (4), the evader is simply subject to the repulsion from the driver, which has the same expression 
than the force that the evader exerts on the driver but with a different (and smaller) coefficient $C_{E}^{D}$.

Finally, both agents are subject to friction forces with the ground, which have the same form, in the opposite direction of the motion: $-\nu_{i} \overrightarrow{v_{i}}$, for $i=d, e$.

The model can be formulated in different ways. The formulation presented in (11) - (5) is based on the general expressions of the attraction-repulsion forces introduced by Gazi \& Passino [22, 23], later widely used in realistic (biological) models [25, 20, 24, 26, 27]. Agents are prevented from collisions by means of strong short-range repulsive forces (large exponents of $\left\|\vec{u}_{e}(t)-\vec{u}_{d}(t)\right\|$ in the denominator), so that, although it is theoretically possible, in practice, the denominators are never (too close to) zero, and the model is in this sense well-posed. Another advantage of this formulation is that each ingredient of the model, especially the circumvention behavior, appears explicitly in the equations, thus facilitating the realistic interpretation of the contribution of each component of the model. For example, the exponent -4 of $\left\|\vec{u}_{d}-\vec{u}_{e}\right\|$ in the circumvention force (which is large compared to the exponent -2 in the attracting force) shows that the circumvention maneuver will only be effective when both agents are sufficiently close to each other.

With this formulation, the control parameter $\kappa(t)$ appears as a factor of the perpendicular component of the force acting on the evader, and, by the simple choice of a value in $\{-1,0,1\}$, practically determines the behavior of the system.

\subsection{Two operating modes, a single control parameter}

Let us define the instantaneous distance between agents $r(t)=\left\|\vec{u}_{d}(t)-\vec{u}_{e}(t)\right\|$.

Then, omitting time-dependence to lighten notation, Eqs. (3)-(4) can be rewritten as

$$
\begin{aligned}
& \dot{\vec{v}}_{d}=-\frac{C_{D}^{E}}{m_{d}} \frac{\vec{u}_{d}-\vec{u}_{e}}{r^{2}}\left[1+\frac{1}{r^{2}}\left(\frac{C_{\mathrm{R}}}{C_{D}^{E}} \delta_{1}^{4}-\delta_{c}^{2}\right)\right]+\kappa \frac{\delta_{1}^{4} \delta_{2}}{r^{3}} \frac{C_{\mathrm{R}}}{m_{d}} \frac{\left(\vec{u}_{d}-\vec{u}_{e}\right)^{\perp}}{r^{2}}-\frac{\nu_{d}}{m_{d}} \vec{v}_{d}, \\
& \dot{\vec{v}}_{e}=-\frac{C_{E}^{D}}{m_{e}} \frac{\vec{u}_{d}-\vec{u}_{e}}{r^{2}}-\frac{\nu_{e}}{m_{e}} \vec{v}_{e} .
\end{aligned}
$$

We have solved the system (11)-(5) numerically with several classical methods (RungeKutta, adaptive or not, Crank-Nicolson), finding that a simple explicit Euler method with time-step of order $10^{-6}$ is sufficiently effective and provides a sufficiently accurate solution for our purposes. Our results are based on exhaustive numerical simulations for a wide range of the parameters preserving the significance of the model. The behavior of the model is stable and coherent under reasonable variations of the parameters. The results we describe here are not dataset dependent. The values we have chosen are those providing the more illustrative figures.

Eq. (6) shows that a necessary condition to have an effective short-range repulsion acting on the driver is therefore that $C_{\mathrm{R}} \delta_{1}^{4}-C_{D}^{E} \delta_{c}^{2}$ is negative, as it is the case for the dataset considered in our study. Similarly, in order to have a faster and more reactive behavior of the driver, we assume that $C_{D}^{E}>C_{E}^{D}$ and that $m_{d}$ and $\nu_{d}$ are sufficiently smaller than $m_{e}$ and $\nu_{e}$ respectively. 
For the numerical simulations and graphical descriptions presented here, we have considered the following dataset for the typical values of the constant parameters of the model: first, $m_{i}$ and $\nu_{i}$ are the mass and the friction coefficients of agent $i$ respectively, with $m_{d}=0.4, m_{e}=1, \nu_{d}=1$ and $\nu_{e}=2$; then, $C_{D}^{E}=3$ is the coefficient of the attractive-repulsive force that the evader exerts on the driver, $C_{E}^{D}=2$ is the coefficient of the repulsive force that the driver exerts on the evader, and $C_{\mathrm{R}}=0.5$ is the coefficient of the circumvention component of the force exerted on the driver when $\kappa= \pm 1$. Finally, the critical distances are as follows: $\delta_{c}=2, \delta_{1}=2$ and $\delta_{2}=2$.

Parameter values are dimensionless (of order one) and chosen so that their relative value allow the system to reproduce the behavior of a realistic driving phenomenon.

The numerical simulations allow us to establish that the system has two operating modes, depending on the value of $\kappa$ :

- Pursuit mode $(\kappa=0)$ : the driver $D$ pursues the evader $E$, which moves away from $D$. Both agents tend to move in the same direction, given by their acceleration vectors, along the straight line $\overline{D E}$. If $\kappa=0$ continuously during a sufficiently long interval of time, both agents' velocities converge asymptotically to the same vector of norm $v_{\text {as }}$ (due to the friction), and the driver stays at a constant distance from the evader, $\delta_{\mathrm{as}}$. See Appendix A for an analytical estimate of these values, and the left panels in Fig. 1: the lower panel shows that, before reaching the constant value $v_{\text {as }} \approx 0.71$, the pursuer is faster than the evader. For the values we have used, $\delta_{\mathrm{as}}=\sqrt{2}$.

- Circumvention mode $(\kappa= \pm 1)$ : the driver $D$ separates from the straight line $\overline{D E}$ and starts a circumvention maneuver around the evader $E$ (clockwise or counterclockwise, depending on the sign of $\kappa$ ). The response of the evader is to move away from the driver. If $\kappa=1$ continuously during a sufficiently long interval of time, as the driver is faster than the evader, the asymptotic behavior of the system is a circular motion of the evader around a fixed point and a circular motion of the driver around the circle described by the evader. See Fig. 1, where the right panels show that, for $t \in[0,80]$, the system tends to a periodic configuration where both agents are separated by a constant distance $\delta_{\text {as }}^{\text {ang }} \approx 1.82$ (not shown in the figure) and have the same angular velocity of norm $\omega_{\text {as }} \approx \pi / 4$ (the period $s$ of the oscillations is $s=2 \pi / \omega_{\text {as }} ;$ bottom-right panel of Fig. 1 shows that $\left.s \approx 8\right)$.

When the driver is sufficiently close to the evader, the circumvention mode is effective and triggers the circular behavior of the agents. See the right panels of Fig. 1, where the driver initial position at $(-6,0)$ is so far from the initial position of the evader $(6,0)$ that, although the control is set to $\kappa=1$ from $t_{0}=0$ to $t_{f}=100$, the first part of the trajectory is almost a straight line. Until $t \approx 32$, both agents are almost still in the horizontal axis: $\vec{u}_{d}=(5,-0.09), \vec{u}_{e}=(9.7,0.02)$. At $t \approx 37, \vec{u}_{d}=(8.62,-0.37)$ and $\vec{u}_{e}=(11.05,0.1)$, the driver is close enough to the evader and the circular behavior becomes perceptible. See the oscillations of $u_{d}^{y}(t)$ and $u_{e}^{y}(t)$, of period $s \approx 40 / 5=8$, in the right-bottom panel of Fig. 1]. 

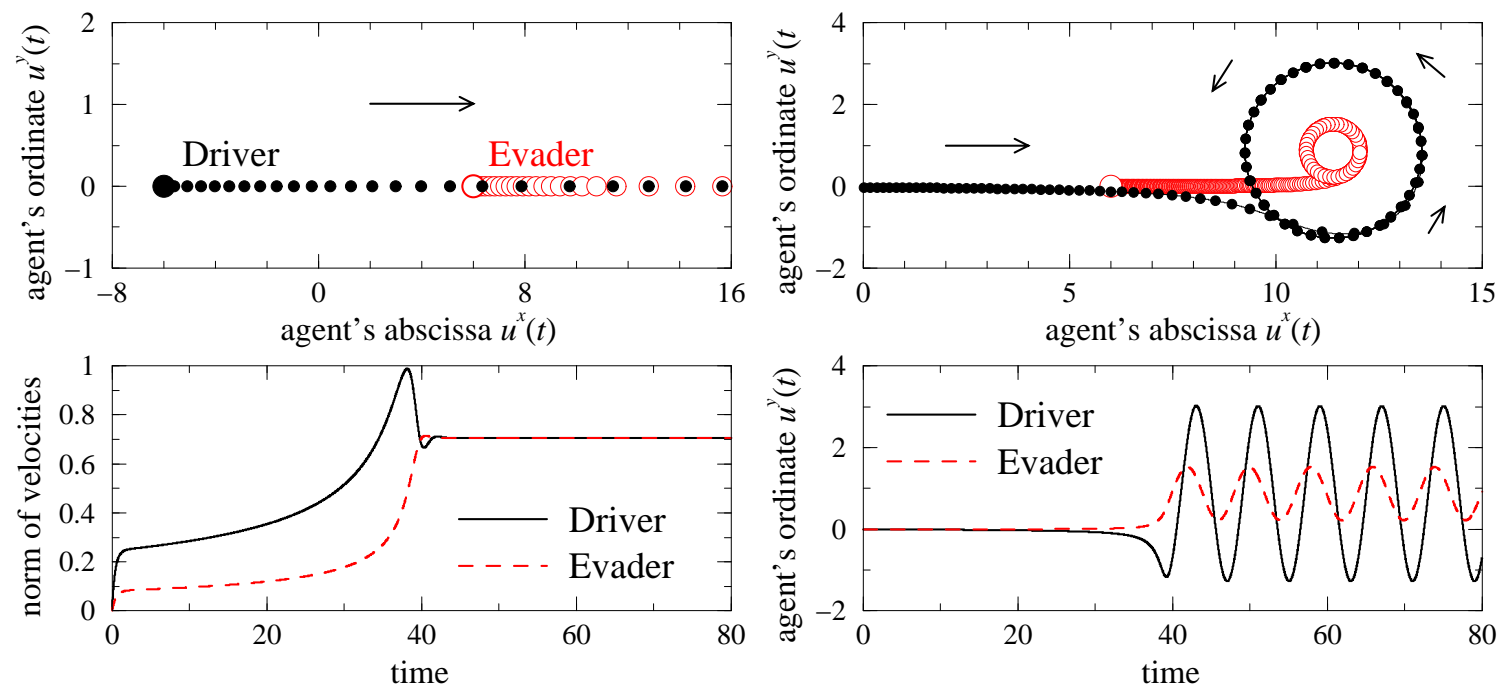

Figure 1: The two operating modes of the system. Left panels: pursuit mode $(\kappa=0)$. Right panels: circumvention mode $(\kappa=1)$. Initial configuration, in both cases: $\vec{u}_{d}=$ $(-6,0), \vec{u}_{e}=(6,0)$ and $\vec{u}_{T}=(1,1)$, with zero initial velocities. Upper panels: agents' trajectories. Arrows denote the direction of motion of the agents. For sufficiently long times, the velocities converge asymptotically to a constant value $v_{s}$. When $\kappa=1$, the driver turns counterclockwise around the evader, which also turns counterclockwise. Leftbottom panel: time variation of the norm of the velocities $\left\|\vec{v}_{d, e}(t)\right\|$, both reaching $v_{\text {as }} \approx$ 0.71 at $t \approx 38.9$. Right-bottom panel: time variation of agents' ordinates: $u_{d}^{y}(t)<0.1$ until $t \approx 32$, while $u_{e}^{y}(t)<0.1$ until $t \approx 37$. For $t>40$, periodic behavior (of period $s \approx 8$ ) with constant angular velocity $\omega_{\text {as }} \approx \pi / 4$ and constant separation $\delta_{\mathrm{as}}^{\text {ang }} \approx 1.82$.

Remark 1: When the driver is far from the evader (i.e., $r \gg 1$ ), the term between brackets in Eq. (6) is such that $1 \gg 1 / r^{2}$, so the first term of this equation (which has coefficient $\left.C_{D}^{E}\right)$ is of order $\mathcal{O}\left(r^{-1}\right)$, while the term with $\kappa(t)$ is of order $\mathcal{O}\left(r^{-4}\right)$. Then, Eq. (6) can be reduced to

$$
\dot{\vec{v}}_{d}=-\frac{C_{D}^{E}}{m_{d}} \frac{\vec{u}_{d}-\vec{u}_{e}}{r^{2}}-\frac{\nu_{d}}{m_{d}} \vec{v}_{d}
$$

Remark 2: In particular, it can be observed that, when $r^{3} \gg \delta_{2}$, then

$$
\left\|\frac{C_{R} \delta_{1}^{4}}{m_{d}} \frac{\vec{u}_{d}-\vec{u}_{e}}{r^{2}}\right\|=\frac{C_{R} \delta_{1}^{4}}{m_{d} r} \gg\left\|\frac{C_{R} \delta_{1}^{4} \delta_{2}}{m_{d}} \frac{\left(\vec{u}_{d}-\vec{u}_{e}\right)^{\perp}}{r^{5}}\right\|=\frac{C_{R} \delta_{1}^{4} \delta_{2}}{m_{d} r^{4}},
$$

so that the term deviating the driver from the pure pursuit trajectory is negligible with respect to the term corresponding to the attracting force exerted by the evader. Therefore, when the driver is sufficiently far from the evader, the value of $\kappa(t)$ has no influence on the behavior of the driver, meaning that $\kappa$ can be set to zero. 


\subsection{On the controllability of the system}

The circumvention mode can be viewed as the active state of the system, where the control parameter $\kappa$ is set to $\mathrm{ON}$, while the pursuit mode is the rest state of the system, where $\kappa$ is set to OFF. With the appropriate combination of both modes, the driver is able to make the evader reach any given target point or move along any (relatively smooth) given trajectory. The resulting behavior of such combination is what we call a driving behavior. See Fig. 2.
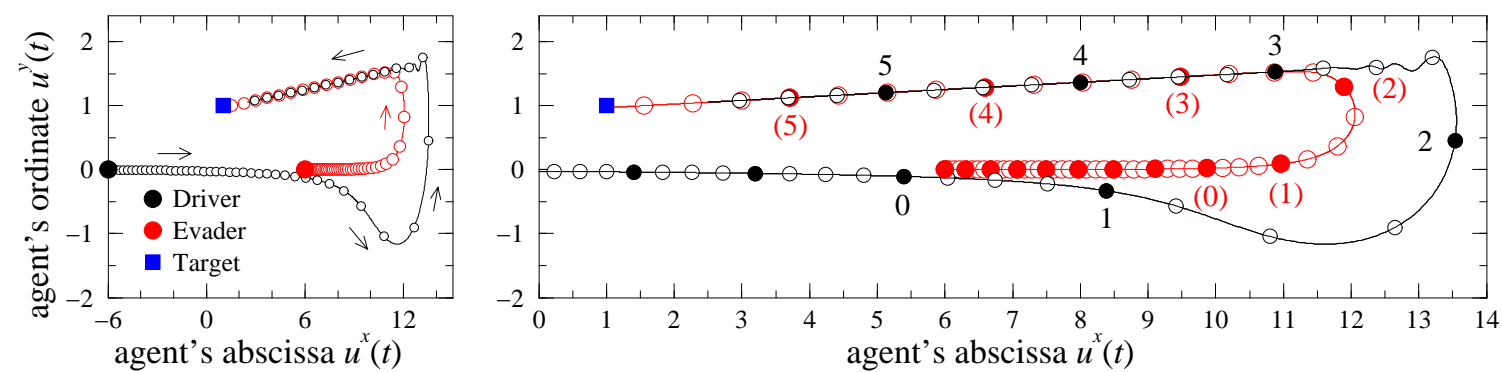

Figure 2: Agents' trajectories (driver: small black circles; evader: large red circles) for an admissible control function $\kappa(t)$. Left: whole trajectories, right: zoom for $u^{x}(t) \in[0,15]$. Symbols are equidistant in time; filled symbols labeled with the same number denote same instant of time (evader's labels are shown in parentheses).

This strongly suggests that the system can be controlled with a single control parameter, $\kappa(t)$. In control theory, a system is said to be fully controllable when, starting from any arbitrary initial state, every possible state of the system can be reached by appropriately adjusting the control parameters (see, e.g., [33]). In this sense, our numerical simulations have shown that agents cannot be separated an arbitrarily long distance, so that the driver-evader system cannot be labelled as fully controllable.

In fact, we prove analytically in Appendix A that, for any initial state, the separation between agents is bounded by above and tends to a distance (of order 1), showing that the evader cannot escape from the driver to infinity, and the driver cannot move away from the evader to infinity. Moreover, numerical simulations show that agents' velocities are also bounded and that, when $\kappa(t)$ remains unchanged for sufficiently long time intervals, both velocities tend asymptotically to the same constant value $\left(v_{\text {as }}\right.$ or $\left.\omega_{\text {as }}\right)$. Finally, agents are prevented from occupying the same place at the same time (i.e., $\vec{u}_{d}(t)=\vec{u}_{e}(t)$ ) by the strong short-range repulsion force that the driver exerts on the evader, as our numerical simulations confirm.

We will say instead that the driver-evader system is partially controllable, in the sense that each agent can be controlled separately: the driver can force the evader to reach any point in the plane (this is shown in the successive sections), and vice-versa, a series of targets for the evader can be selected so that the driver is driven to reach any point in the plane.

The question arises now as how the driver-evader system behavior can be optimized to minimize a given cost functional accounting for the use of the lateral propellers. 


\section{Optimal control: two optimal open-loop controls, one feedback control law}

Denote by $B_{\rho}(T)$ a ball of radius $\rho$ centered in $T$, assume that the evader is initially far from the driver (e.g., $r\left(t_{0}\right)=\left\|\vec{u}_{d}\left(t_{0}\right)-\vec{u}_{e}\left(t_{0}\right)\right\| \gg \delta_{1}$ ), and assume also that $D, E$ and the ball $B_{\rho}(T)$ are not aligned, that is, $\overline{D E} \cap B_{\rho}(T)=\varnothing$.

Consider now the objective of driving the evader $E$ into the target ball $B_{\rho}(T)$ at a final time $t_{f}$ by controlling the driver $D$ with an appropriate strategy $\kappa(t)$. As $D, E$ and $B_{\rho}(T)$ are not aligned, the circumvention mode has to be activated and/or has to remain active a suitable time to modify the trajectory of the evader and guide it towards the target ball $B_{\rho}(T)$.

Such an objective must appraise the cost of 1) forcing the system to leave its resting state, and/or 2) keeping the system in an active state. This cost is given by the functional

$$
J(\kappa) \stackrel{\text { def }}{=} \sigma_{1} N_{\mathrm{ig}}(\kappa)+\sigma_{2} \mathcal{C}(\kappa),
$$

where $N_{\text {ig }}(\kappa)$ is the number of times that the system is forced to leave its resting state in the time interval $\left[t_{0}, t_{f}\right]$ (that is, the number of ignition processes, where $\kappa(t)$ changes from 0 to \pm 1 ), and $C(\kappa)$ is the total time during which the system is active in $\left[t_{0}, t_{f}\right](i . e$, the time spent with a lateral propeller in active mode, where $\kappa(t)$ has a non zero value),

$$
\mathcal{C}(\kappa)=\int_{t_{0}}^{t_{f}}|\kappa(t)| d t
$$

and $\sigma_{1,2}$ are nonnegative weights fixed to balance the contribution of each partial cost.

An optimal control problem $(O C P)$ can then be formulated as

$$
(O C P)\left\{\begin{array}{l}
\operatorname{Min} J(\kappa)=\sigma_{1} N_{\mathrm{ig}}(\kappa)+\sigma_{2} \mathcal{C}(\kappa) \\
\kappa \in U_{\mathrm{ad}}=\left\{\kappa:\left[t_{0}, t_{f}\right] \rightarrow\{-1,0,1\} \text { such that } \vec{u}_{e}\left(t_{f}\right) \in B_{\rho}(T)\right\}
\end{array},\right.
$$

where $U_{\text {ad }}$ is the set of admissible controls.

Here we solve $(O C P)$ for $N_{\text {ig }}=0$ and $N_{\text {ig }}=1$ in sections 3.1 and 3.2 respectively, finding the corresponding optimal open-loop controls. Our results show that the system is highly sensitive to small variations of the conditions of the problem, so that a general open-loop control for $N_{\text {ig }}>1$ would not be of practical interest. Instead, we provide in section 3.3 a feedback control law for $N_{\text {ig }}>1$ that substantially reduces the cost of the open-loop controls for $N_{\mathrm{ig}} \leq 1$, and preserves the number of ignitions at a relatively low value $\left(N_{\text {ig }}=4\right.$; see later $)$.

\subsection{Optimal open-loop guidance strategies for $N_{\mathrm{ig}} \leq 1$}

Solving $(O C P)$ for $N_{\text {ig }}=0$ consists in minimizing $\mathcal{C}(\kappa)$ for $\kappa\left(t_{0}\right)=\kappa_{0}$, with $\kappa_{0}= \pm 1$. Indeed, if $\kappa\left(t_{0}\right)=0$, then $\kappa \notin U_{\mathrm{ad}}$, because $D$ and $E$ are initially not aligned with the target ball and no inition process can be used to modify the trajectory of the evader, 
so $\vec{u}_{e}(t) \notin B_{\rho}(T)$ for all $t \in\left[t_{0},+\infty\right)$. Similarly, solving $(O C P)$ for $N_{\text {ig }}=1$ consists in minimizing $\mathcal{C}(\kappa)$ for $\kappa(t)$ of the form $\kappa(t)=\kappa_{0}$ in a time interval $\left[t_{\mathrm{ON}}, t_{\mathrm{OFF}}\right]$ and $\kappa(t)=0$ outside.

We thus seek two control function profiles like those sketched in Fig. 3 ,
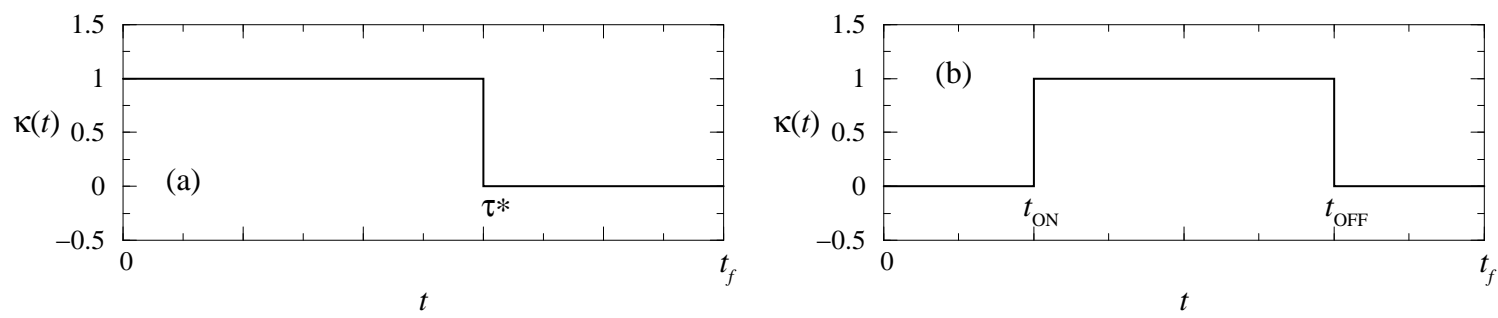

Figure 3: Profiles of the control function $\kappa(t)$ with $\kappa_{0}=1$ for each case (a) $N_{\text {ig }}=0$ and (b) $N_{\text {ig }}=1$.

\subsection{1 $\quad N_{\text {ig }}=0$ :}

The following result holds:

Let $\kappa_{\tau}(t): \mathbb{R} \rightarrow\{-1,0,1\}$ be the following step-function in the time interval $\left[t_{0}, t_{f}\right]$,

$$
\kappa_{\tau}(t)= \begin{cases}\kappa_{0} & \text { if } t<\tau \\ 0 & \text { if } t \geq \tau\end{cases}
$$

where $\kappa_{0}= \pm 1$ is the initial value at time $t_{0}: \kappa_{\tau}\left(t_{0}\right)=\kappa_{0}$. Then, if $t_{f}$ is sufficiently large, there exists an interval $\left[\tau_{\alpha}, \tau_{\omega}\right] \subset\left(t_{0}, t_{f}\right)$ such that, for all $\tau \in\left[\tau_{\alpha}, \tau_{\omega}\right]$, there exists a time $t \in\left(t_{0}, t_{f}\right)$ for which the evader is in the interior of the ball of radius $r$ centered in the target $T$. That is:

$$
\forall \tau \in\left[\tau_{\alpha}, \tau_{\omega}\right], \exists t \in\left(t_{0}, t_{f}\right) \text { such that }\left\|\vec{u}_{e}(t)-\vec{u}_{T}\right\|<r \text {. }
$$

Moreover, if $r \rightarrow 0$, then the interval $\left[\tau_{\alpha}, \tau_{\omega}\right]$ shrinks to a single point $\tau^{*}$ such that there exists a time $t \in\left(t_{0}, t_{f}\right)$ for which $\vec{u}_{e}(t)=\vec{u}_{T}$. See $\tau^{*}$ in Fig. [3(a).

The proof is based on a continuity argument applied to a (numerical) shooting method.

Fig. 4 shows the trajectories of the evader for different values of $\tau$. By a continuity argument, it is possible to find the values of $\tau_{\alpha}, \tau_{\omega}$ and $\tau^{*}$ with a simple shooting method based on comparing the direction of the velocity vector of the evader $\vec{v}_{e}$ with respect to the direction towards the target when the control is switched off. See Appendix B for a more detailed description of the decision test of the shooting method.

In this case, the cost of a strategy $\kappa_{\tau}(t)$ given by (13) is $\mathcal{C}\left(\kappa_{\tau}\right)=\tau$ for all $\tau \in\left[\tau_{\alpha}, \tau_{\omega}\right]$, so that the optimal strategy is $\kappa_{\tau_{\alpha}}(t)$. For a sufficiently small value of $r$ (we used $r=10^{-4}$ in Fig. 4), the interval $\left[\tau_{\alpha}, \tau_{\omega}\right]$ colapses to the value $\tau^{*}$ and the cost of the optimal control is $\mathcal{C}\left(\kappa_{\tau}^{*}\right)=\tau^{*}$.

Noticeably, Fig. 4 reveals that the system is highly sensitive to small variations of $\tau$ around the optimal value $\tau^{*}$ : the same variation of $\tau$ produces a larger variation of the 


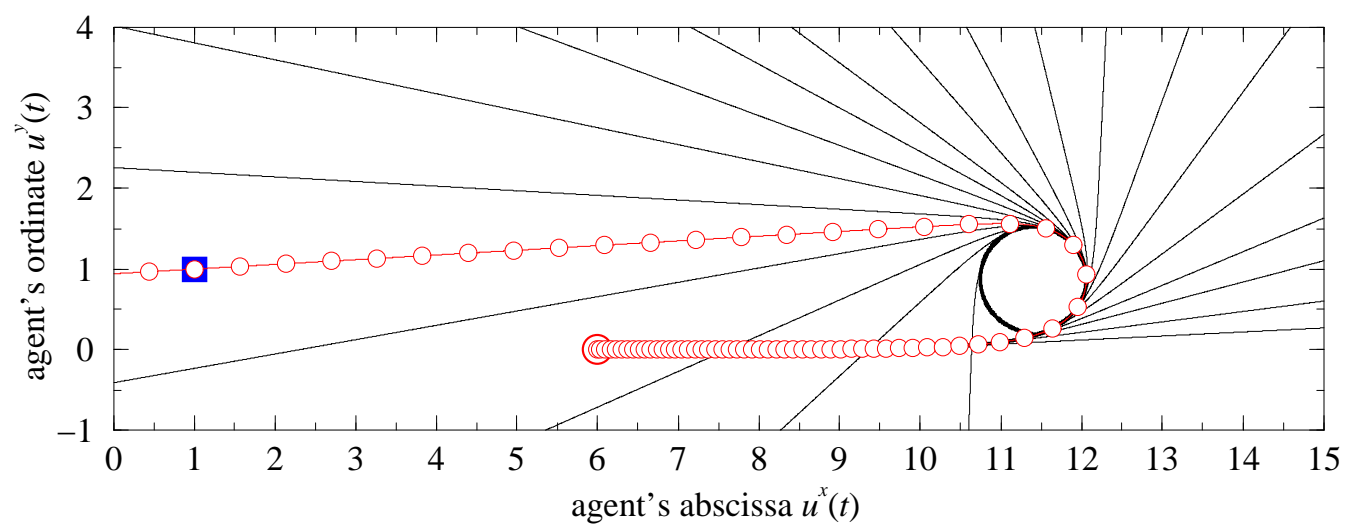

Figure 4: Shooting method for finding the optimal value $\tau^{*}$ of the control function shown in Fig. 3(a), for which $\left\|\vec{u}_{e}(t)-\vec{u}_{T}\right\|<\rho$ for some $t \in\left(0, t_{f}\right)$ (here we used $\rho=10^{-4}$ ). Filled (blue) square denotes target's position $\vec{u}_{T}=(1,1)$, empty (red) circle evader's initial position $\vec{u}_{e}(0)=(6,0)$; driver's initial position $\vec{u}_{d}(0)=(-6,0)$ is not depicted. Line with (red) circles denote the trajectory of the evader for the optimal value $\tau^{*}=41.15$. Wide (black) line denotes the accumulation circle around which the evader turns if the control is kept to one. Thin (black) lines denote trajectories of the evader for the following non-equispaced values of $\tau: 34,36,37,37.5,38,38.4,38.7,39,39.3,39.6,39.9,40.2,40.4$, 40.6, 40.8, 41, 41.3, 41.6, 42 and 43.

deviation of $\vec{v}_{e}$ with respect to a reference line (e.g., the horizontal line) when $\tau$ is close to $\tau^{*}$ than when $\tau$ is far from $\tau^{*}$ : for $\Delta \tau=2$, the angular variation $\theta$ from $\tau=39.9$ to 42 is larger than $\pi / 2$ (actually, $\theta_{42}-\theta_{39.91} \approx 1.62$ ), and is 10 times smaller from $\tau=34$ to $36\left(\theta_{36}-\theta_{34} \approx 0.16\right)$.

\subsection{2 $\quad N_{\text {ig }}=1:$}

In the previous case, the optimal control is set to 1 in the whole interval $\left[0, \tau_{\alpha}\right)$. The possibility of using one ignition process allows us to consider controls where the system is at rest while the driver is approaching the evader, taking advantage of the fact that when the driver is far from the evader the circumvention term has (practically) no influence on the trajectory of the driver (and therefore, on the behavior of the system); see Remark 2.

This delay in the ignition process substantially reduces the cost by allowing the use of a control function profile like the one depicted in Fig. 3(b), defined by

$$
\kappa_{t_{\mathrm{ON}}}^{\mathrm{toF}}(t)=\left\{\begin{array}{ll}
\kappa_{0} & \text { if } t_{\mathrm{ON}} \leq t<t_{\mathrm{OFF}} \\
0 & \text { elsewhere }
\end{array},\right.
$$

where $t_{\mathrm{ON}}$ and $t_{\mathrm{OFF}}$ are the instants of time in which the control is switched on $\left(\kappa=\kappa_{0}=\right.$ $\pm 1)$ and off $(\kappa=0)$ respectively. For these control functions, the cost is given by

$$
\mathcal{C}\left(\kappa_{t_{\mathrm{ON}}}^{t_{\mathrm{OFF}}}\right)=\int_{t_{0}}^{t_{f}}\left|\kappa_{t_{\mathrm{ON}}}^{t_{\mathrm{OFF}}}(t)\right| d t=t_{\mathrm{OFF}}-t_{\mathrm{ON}},
$$


so that the $(O C P)$ problem is reduced to find the values of $t_{\mathrm{ON}}$ and $t_{\mathrm{OFF}}$ minimizing $t_{\mathrm{OFF}}-t_{\mathrm{ON}}$.

These values are found by means of two successive (numerical) shooting methods. Let us consider the extreme case where $\rho=0$ (numerically we used $\rho=10^{-8}$ ), so that the solution of the shooting method described in the previous section for $N_{\text {ig }}=0$ is unique.

The first shooting method consists in finding, for each value of $t_{\mathrm{oN}}$, the (unique) value $t_{\mathrm{OFF}}^{*}\left(t_{\mathrm{ON}}\right)$ for which there exists a time $t \in\left(t_{0}, t_{f}\right)$ such that $\vec{u}_{e}(t)=\vec{u}_{T}$. This shooting method is the one presented in the previous case where $N_{\text {ig }}=0$. In fact, in that case, $t_{\mathrm{ON}}=0$. This procedure allows us to build a function $t_{\mathrm{OFF}}^{*}\left(t_{\mathrm{ON}}\right)$ in such a way that the function $\kappa_{t_{\mathrm{ON}}}^{t_{\mathrm{OF}}^{*}}(t)$ is admissible: $\kappa_{t_{\mathrm{ON}}}^{t_{\mathrm{OF}}^{*}} \in U_{\mathrm{ad}}$.

Then, the second shooting method consists in finding the value of $t_{\mathrm{ov}}$ for which the characteristic function of the interval $\left[t_{\mathrm{ON}}, t_{\mathrm{OFF}}^{*}\left(t_{\mathrm{ON}}\right)\right]$ minimizes $\mathcal{C}\left(\kappa_{t_{\mathrm{ON}}}^{t_{\mathrm{OF}}}\right)$.
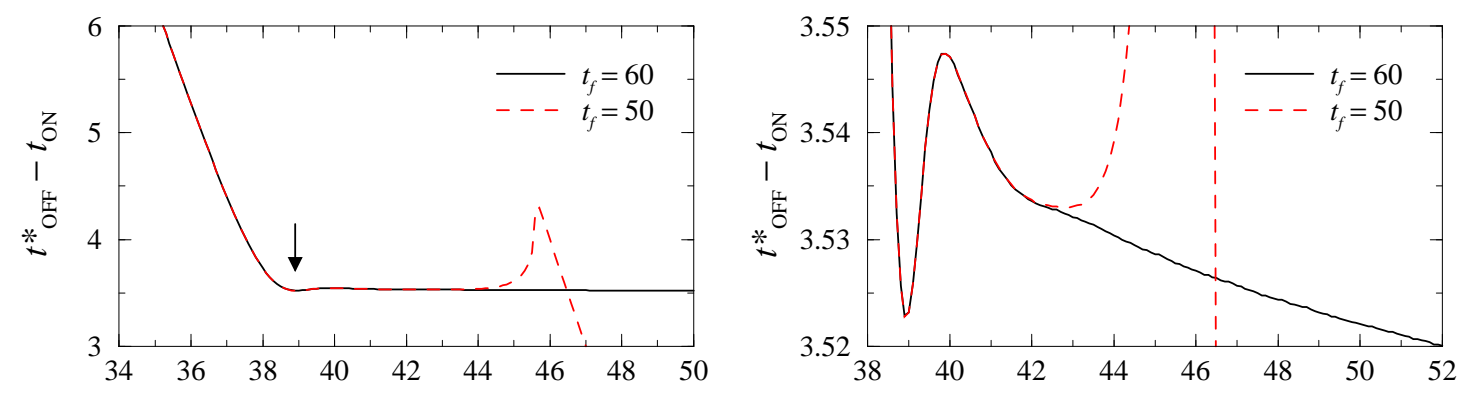

Figure 5: Shooting method to find the optimal values $t_{\mathrm{ON}}^{*}$ and $t_{\mathrm{OFF}}^{*} t_{\mathrm{ON}}$ or which $\left\|\vec{u}_{e}(t)-\vec{u}_{T}\right\|<$ $\rho$ for some $t \in\left(0, t_{f}\right)\left(\rho=10^{-8}\right)$, for two different values of $t_{f}$ : solid (black) line, $t_{f}=60$; dashed (red) line, $t_{f}=50$. Left panel: cost function $\mathcal{C}\left(t_{\mathrm{ON}}\right)=t_{\mathrm{OFF}}^{*}\left(t_{\mathrm{ON}}\right)-t_{\mathrm{ON}}$, exhibiting a plateau starting at $t_{\mathrm{ON}}^{*} \approx 38.92$ (arrow) and situated at $\mathcal{C}^{*} \approx 3.52$. Right panel: zoom of the vertical axis, revealing the nonlinear shape of the curve with a minimum at $t_{\mathrm{0N}}^{*}$, closely followed by a local maximum at $t_{\mathrm{ON}} \approx 39.82$, and a decreasing regime for $t_{f}$ sufficiently large. The relative amplitude of the nonlinearity is $\approx 7 \times 10^{-3}$.

Fig. 5 shows the cost function $\mathcal{C}$ as a function of $t_{\mathrm{ON}}: \mathcal{C}\left(t_{\mathrm{ON}}\right)=t_{\mathrm{OFF}}^{*}\left(t_{\mathrm{ON}}\right)-t_{\mathrm{ON}}$.

When $t_{f}$ is sufficiently large, the $\operatorname{cost} \mathcal{C}\left(t_{\text {oN }}\right)$ tends to a constant value $\mathcal{C}^{*} \approx 3.52$ which is the time is takes to the driver to make the evader turn back towards the target. See Fig. 6, where two examples with different ignition times $t_{\mathrm{ON}}$ yield (approximately) the same cost $\mathcal{C}^{*}$.

This value $\mathcal{C}^{*}$ constitutes a substantial reduction of the optimal cost found for $N_{\text {ig }}=0$.

In fact, Fig. 5 shows that there is a wide range of values of $t_{\mathrm{ON}}$ yielding a similar value of the cost, so that, for a tolerance larger than $10^{-2}$, the optimal control would not be unique. This would allow the system to accept another criteria or another restriction to determine the optimal strategy, which can be for instance to minimize the total time or the total distance travelled by the agents (and which would be equivalent to consider the cost of back-propellers).

However, if the variations of $\mathcal{C}^{*}$ of order $10^{-3}$ are relevant, a closer analisys shows that the first linear decreasing range in Fig. 5, which lasts until $t_{\mathrm{0N}}^{*} \approx 38.9$, is due to the fact that during this interval of time $\left[0, t^{*}\right]$ the driver is far from the evader, that is, 

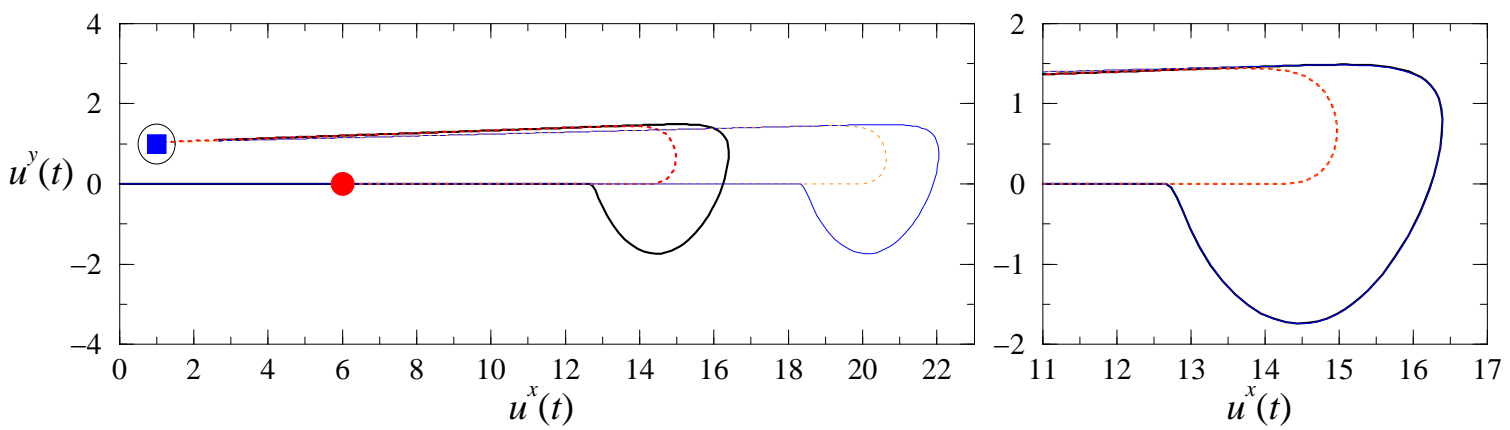

Figure 6: Agents' trajectories for two ON-OFF controls yielding the same cost $\mathcal{C}^{*} \approx 3.53$ in Fig. 5. Wide lines (short trajectories, black and red): $t_{\mathrm{ON}}=42, t_{\mathrm{OFF}}^{*}=45.5337$; thin lines (long trajectories, blue and orange): $t_{\mathrm{ON}}=50, t_{\mathrm{OFF}}^{*}=53.5221$. Solid lines denote the driver, dotted lines the evader. In the short trajectory the evader reaches the target at $t_{f}=65$, while it must go until $t_{f}=81$ in the long trajectory. In both cases the turning back maneuver takes the same time $\mathcal{C}^{*}$ and has the same shape. Right panel: trajectories during the turning back maneuver overlap when shifted $t=t-5.64$.

$r=\left\|\vec{u}_{d}-\vec{u}_{e}\right\| \gg 1$, so that the term with $\kappa$ in Eq. (3) can be neglected (it is of order $\mathcal{O}\left(r^{-4}\right)$ ) with respect to the terms corresponding to the attracting force (of order $\mathcal{O}\left(r^{-2}\right)$ ). This means that switching on the control before $t_{\mathrm{ON}}^{*}$ doesn't contribute effectively to reach the target and is a waste of resources. In fact, the optimal value of the cost is reached at $t_{\mathrm{ON}}^{*}: \mathcal{C}(38.9)=3.5228$. When $t_{f}$ is so short that the turning back maneuver can not be completed, then $t_{\mathrm{OFF}}^{*}\left(t_{\mathrm{ON}}\right)=t_{f}$, so that the cost function $\mathcal{C}\left(t_{\mathrm{ON}}\right)$ decreases (again linearly with $t_{\mathrm{ON}}$ ) to zero (but of course the target is not reached). Note that, for all $t_{\mathrm{oN}}$, the value $t_{\mathrm{OFF}}^{*}\left(t_{\mathrm{ON}}\right)$ will exist provided $t_{f}$ is sufficiently large.

Interestingly, $\mathcal{C}\left(t_{\mathrm{oN}}\right)$ exhibits an abrupt nonlinearity of small amplitude at $t_{\mathrm{oN}}^{*}$; see the right panel of Fig. 5. In a pure pursuit regime, the velocity of the agents both converge to a constant value $v_{\text {as }}$ due to the friction with the ground. See the lower left panel in Fig. 1, The nonlinearity is located precisely at the time where the evader reaches this constant velocity $\left(v_{\mathrm{as}} \approx 0.71\right)$. The curve of $\mathcal{C}\left(t_{\mathrm{oN}}\right)$ reaches a (local) maximum at a value of $t_{\mathrm{ON}}$ slightly larger than $t_{\mathrm{ON}}^{*}$, due to that the driver is close to the evader and both agents' velocities have converged to $v_{\mathrm{as}}$. For larger values of $t_{\mathrm{0N}}$ in the horizontal plateau of Fig. 5, the turning back maneuver is practically identical in space and time; see Fig. 6, especially the right panel, where we show that two different values of $t_{\mathrm{ON}}$ in the plateau yield two turning back maneuvers that practically overlap. The slight decrease of the plateau shown in the right Panel of Fig. 5 is due to the fact that turning back maneuvers are less consuming the farther from the target they takes place, because the angle at which the control is switched off is smaller. This situation is reversed (that is, the plateau increases) when the target point is above the turning back region -e.g., at $(x, y)=(1,2)$.

Although the relative amplitude of the nonlinearity of $\mathcal{C}\left(t_{\mathrm{oN}}\right)$ is very small $(0.024$ with respect to 3.52), it unexpectedly adds an important complexity to the study of the system: the minimum located at $t_{\mathrm{ON}}^{*}$ can be global or local depending on $t_{f}$, therefore complicating 
the search for optimal directions of descent in numerical minimization methods.

Moreover, the high sensitivity of the system detected in the previous section is also in action if control functions like $\kappa_{t_{\mathrm{ON}}}^{t_{\mathrm{OF}}^{*}}(t)$ are used when small variations of $t_{\mathrm{OFF}}^{*}$ with respect to $t_{\text {on }}$ can occur.

This section shows that, despite a quite predictive general behavior of the system (no signs of chaos have been detected), its high sensitivity strongly suggests the use of closed-loop controls.

\subsection{A feedback control law for $N_{\text {ig }}>1$}

For systems that are subject to conditions of high sensitivity like those described in section 3.1, closed-loop or feedback controls offer the possibility of correcting instantaneously the state of the system for deviations from the desired behavior [31, 32]. Moreover, the explicit form of the control as a function of time need not to be known a priori in the whole time interval $\left[t_{0}, t_{f}\right]$. In turn, feedback laws have to pay the cost of continuously monitoring the position and velocity of the agents.

We present here a feedback control law based on the following observations:

1. In real situations, the orientation of the vector $\vec{v}_{e}(t)$ used in the shooting method can be difficult to observe with the accuracy required by the high sensitivity of the system.

Instead, the alignment $a(t)$ of the driver $D$ and the evader $E$ with the target point $T$ is easier to observe and is a good approximation of $\vec{v}_{e}(t)$.

2. When the driver is sufficiently far from the evader, $\kappa(t)$ can be set to zero (Remarks 1 \& 2).

The instantaneous information about the state of the system is processed in real time to determine the distance separating both agents $r(t)$ and the alignment $a(t)$. The alignment $a(t)$ can be charaterized by the following scalar product (time dependence is omitted to lighten notation):

$$
a(t)=\left(\vec{u}_{T}-\vec{u}_{d}\right) \cdot\left(\vec{u}_{e}-\vec{u}_{d}\right)^{\perp}=\left(u_{T}^{x}-u_{d}^{x}\right)\left(u_{d}^{y}-u_{e}^{y}\right)+\left(u_{T}^{y}-u_{d}^{y}\right)\left(u_{e}^{x}-u_{d}^{x}\right) .
$$

The sign of $a(t)$ reveals in which half-plane the target $T$ is with respect to the line $\overline{D E}$, and can be used to determine the sign of $\kappa(t)$.

Moreover, $|a(t)|$ is an instantaneous measure of how urgently the control must be set to ON. Let us consider a maximal tolerance of deviation $\bar{a}$. The feedback control law is based on the idea that when $|a(t)|$ is smaller than $\bar{a}$, it is possible to consider that $T$ is practically on the line $\overline{D E}$, so that $\kappa(t)$ can be set to OFF, thus saving cost, and when $|a(t)|>\bar{a}$, the deviation is excessive and the control must be set to ON. The tolerance of deviation $\bar{a}$ is an effective bound for both the angle and the intensities of the velocities $\left(a=\left\|\vec{u}_{T}-\vec{u}_{d}\right\|\left\|\vec{u}_{e}-\vec{u}_{d}\right\| \cos \left(\vec{u}_{T}-\vec{u}_{d}, \vec{u}_{e}-\vec{u}_{d}\right)^{\wedge}\right)$, so $|a|<\bar{a}$ restricts also the velocities of the agents: a slightly deviated evader at a high speed can miss the target as well as a largely deviated evader at a lower speed. 
Note also that when $|a(t)|<\bar{a}$, the control can be switched off provided the evader and the target are at the same side with respect to the driver, in order to prevent the driver from driving the evader away from the target; that is, $\kappa(t)$ can be set to zero only if the scalar product $\left(\vec{u}_{e}-\vec{u}_{T}\right) \cdot\left(\vec{u}_{e}-\vec{u}_{d}\right)$ is negative.

Finally, Remark 2 is introduced into the feedback law by means of the characteristic function

$$
\mathcal{X}(t)= \begin{cases}0 & \text { if } r^{3}(t) \gg \delta_{2} \\ 1 & \text { if not }\end{cases}
$$

which serves to switch off the control when the driver is far $\left(r^{3}(t) \gg \delta_{2}\right)$ from the evader.

The feedback control law can then be written as follows:

$$
\kappa_{\mathrm{F}}(t)=\mathcal{X}(t) \times\left\{\begin{array}{cl}
0 & \text { if }|a(t)| \leq \bar{a} \text { and }\left(\vec{u}_{e}-\vec{u}_{T}\right) \cdot\left(\vec{u}_{e}-\vec{u}_{d}\right)<0 \\
\operatorname{sign}\{a(t)\} & \text { if }|a(t)|>\bar{a} \text { or }\left(\vec{u}_{e}-\vec{u}_{T}\right) \cdot\left(\vec{u}_{e}-\vec{u}_{d}\right) \geq 0 .
\end{array}\right.
$$

We have solved the system (11) -(5) numerically using the condition $r^{3}(t)>3 \delta_{2} / 2$ to have $\mathcal{X}(t)=0$ in expression (19). We have considered an alignment tolerance $\bar{a}=4 \times 10^{-1}$. The rest of values are as in previous sections. Let us refer to this case as case (a).

The result is that the feedback law reduces substantially the cost obtained with the open-loop control in Sec. 3.1: $\mathcal{C}_{\mathrm{F}}^{(\mathrm{a})}=1.43$, so an improvement of $60 \%$ with respect to $\mathcal{C}^{*}=3.53$, with however a slight increase of the number of ignition processes, from $N_{\mathrm{ig}}^{*}=1$ to $N_{\text {ig }}^{(\mathrm{a})}=4$.

See Figs. 7, 9 and [8(a1),(a2).
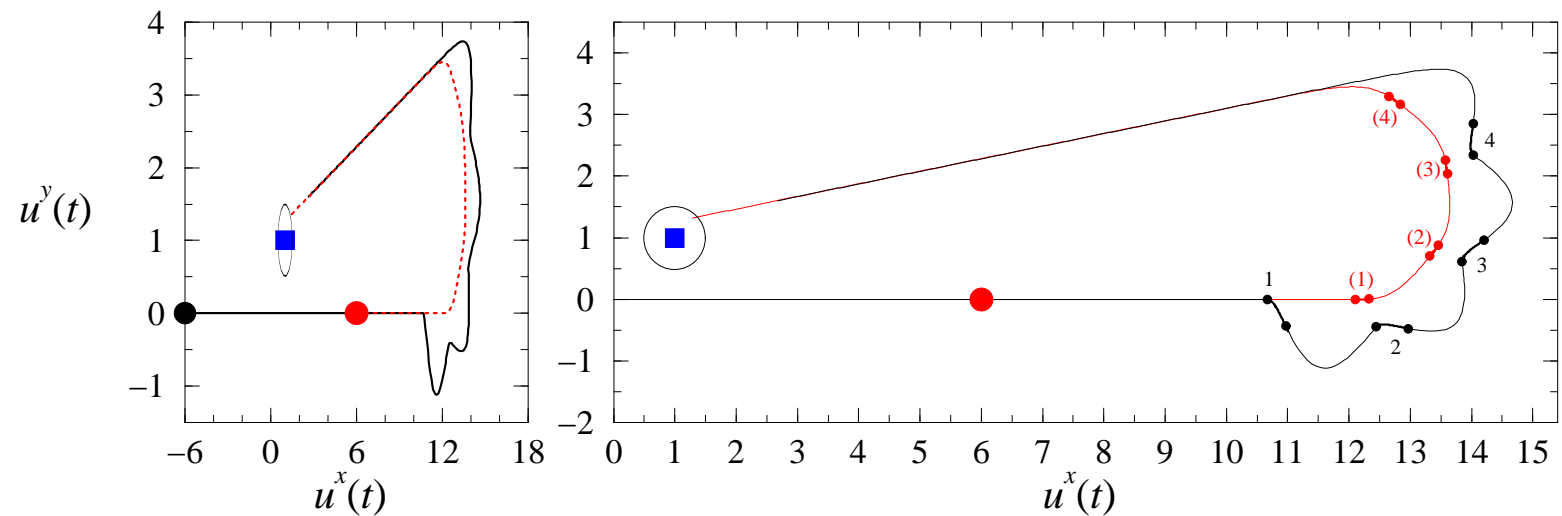

Figure 7: Agents' trajectories for the feedback law $\kappa_{\mathrm{F}}(t)$ given in (19), with alignment tolerance $\bar{a}=4 \times 10^{-1}$. Left, whole trajectories; right, detail. Wide solid segments denote intervals of time with active control (ON). Small solid circles correspond to onsets and ends of such intervals. Numbers denote segments of the same interval of time: $S_{1}=$ $[39.17,39.55], S_{2}=[41.54,41.89], S_{3}=[43.77,44.11]$ and $S_{4}=[45.98,46.32]$. The cost is $\mathcal{C}_{\mathrm{F}}^{(\mathrm{a})}=1.43$.

Figs. 7 shows the trajectories of the agents (the whole trajectories in the left panel, and a zoom of $u^{x}(t) \in[0,15]$ in the right panel). Surprisingly, the trajectory of the driver 
exhibits an oscillatory behavior around the circular trajectory of the evader, allowing the driver to remain closer to the evader than in the previous cases (see, e.g., Fig. 6). Moreover, the time spent with the control in active mode $\left(\kappa_{\mathrm{F}}=1\right)$ is surprisingly short compared to the time spent in this state in the open-loop control $\kappa_{t_{\mathrm{ON}}}^{t_{\mathrm{OF}}}(t)$.
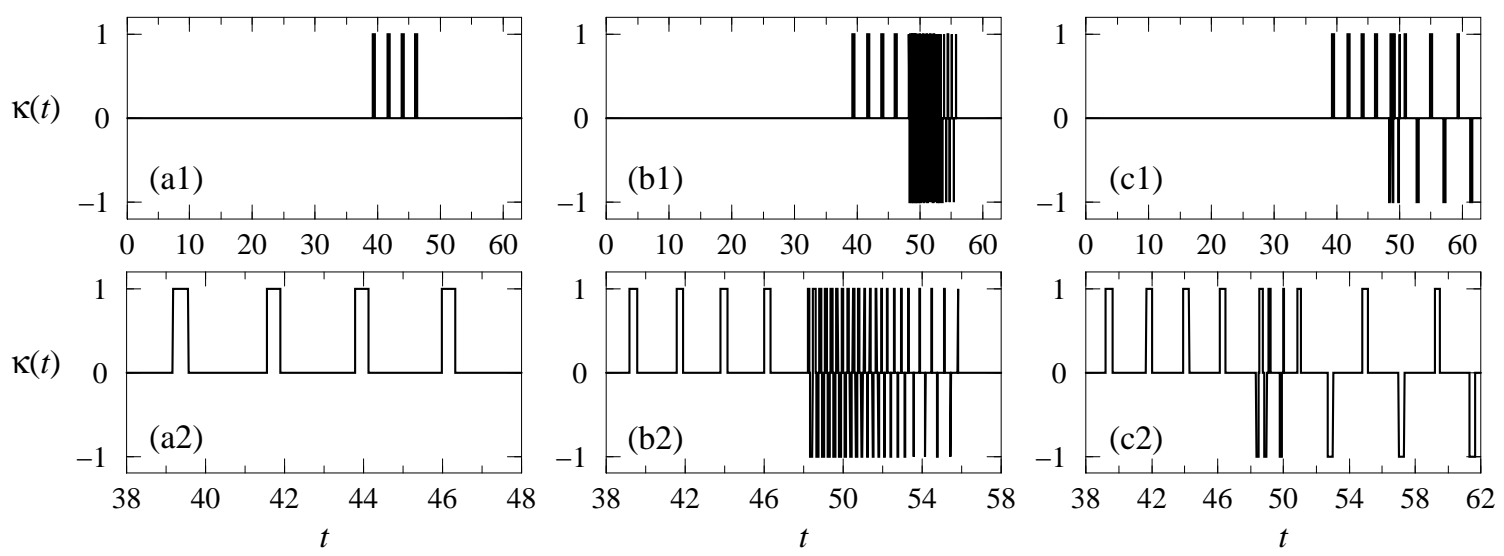

Figure 8: Feedback control laws resulting from the three previous situations denoted (a), (b) and (c) in Figs. 7] and 9, showing the extreme sensitivity of the system under variations of the alignment $a^{*}$ and the switching times of the control $\kappa(t)$. Lower panels (abc2): zoom of upper panels (abc1). Resulting costs: $\mathcal{C}_{\mathrm{F}}^{(\mathrm{a})}=1.43, \mathcal{C}_{\mathrm{F}}^{(\mathrm{b})}=4.275$ and $\mathcal{C}_{\mathrm{F}}^{(\mathrm{c})}=4.13$. Number of ignition processes are $N_{\mathrm{ig}}^{(\mathrm{a})}=4, N_{\mathrm{ig}}^{(\mathrm{b})}=43$ and $N_{\mathrm{ig}}^{(\mathrm{c})}=16$.

The improvement consists in that the circumvention mode is interrupted $\left(\kappa_{\mathrm{F}}=0\right)$ during the surrounding motion of the driver around the evader, reducing the time spent with $\kappa_{\mathrm{F}}=1$ to four small intervals $S_{j}, j=1, \ldots, 4$, of total length $\mathcal{C}_{\mathrm{F}}^{(\mathrm{a})}=1.43$, and, accordingly, $N_{\mathrm{ig}}^{(\mathrm{a})}=4$. See the wide solid segments in the trajectories of the agents in Fig. 7 and Figs $8(\mathrm{a} 1)$ and (a2), which show the resulting control function $\kappa_{\mathrm{F}}(t)$ for $t \in\left[t_{0}, t_{f}\right]$, with $t_{0}=0$ and $t_{f}=63$.

The solution found in Fig. 7 with the feedback law (19) can indeed be considered a good solution of the control problem. However, this is not a general situation, as shown by the wide range of cases analysed in our numerical simulations, because of the high sensitivity exhibited by the system. Let us illustrate this observation here by showing the results for two slightly different external conditions; see the cases (b) and (c) in Figs. 8 and 9 .

Case (b) and (c) use an alignment tolerance $\bar{a}=10^{-1}$. Fig. 9 shows that the evader follows almost the same trajectory than in case (a) (depicted in the figure to facilitate the comparison) and reaches the target with a more accurate orientation. However, such a small deviation requires an enormous increase of the use of the lateral propellers, as shown in Fig. 8)(b2). Not only the cost of having the control set to 1 is larger, $\mathcal{C}_{\mathrm{F}}^{(\mathrm{b})}=4.275$ (and larger than with the open-loop controls), but a much greater number of ignition processes is required $\left(N_{\mathrm{ig}}^{(\mathrm{b})}=43\right)$, moreover involving both the right and the left propellers alternatively (i.e., $\kappa_{\mathrm{F}}=+1,0,-1,0,+1, \ldots$ ). 

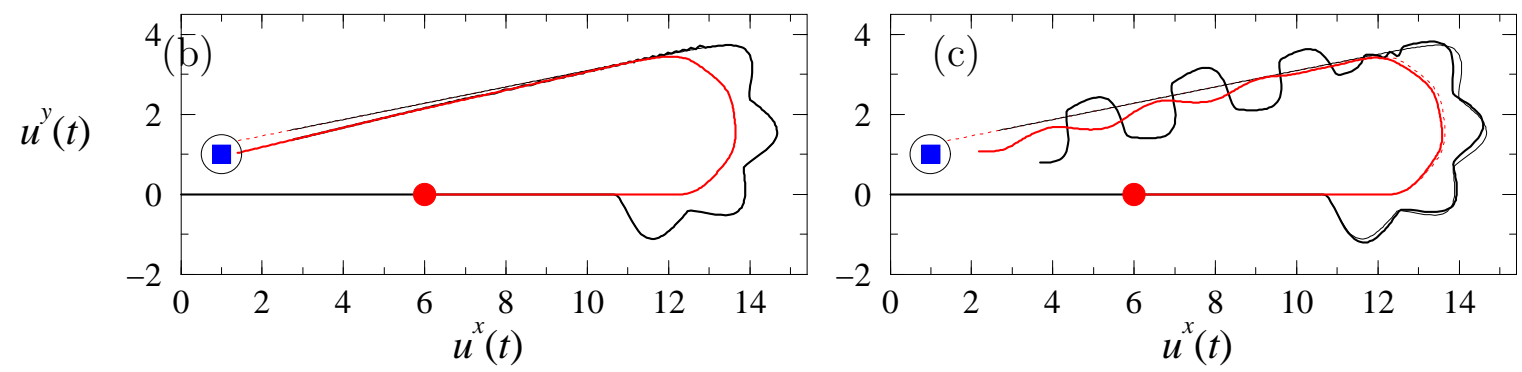

Figure 9: Perturbations of the previous case depicted in Fig. 7 , (a) perturbation of the alignment tolerance: here $\bar{a}=10^{-1}$ instead of $4 \times 10^{-1}$, and (b) perturbation of relative order $10^{-3}$ of the first interval of activation: here $S_{1}^{\prime}=[39.17,39.6]$ instead of $S_{1}=[39.17,39.55]$. For $t \geq 39.6$ the same feedback law (19) is used. In (c) the target is not reached because $t_{f}$ is too small; for a larger $t_{f}$, the target is reached with no additional cost $\left(\kappa_{\mathrm{F}}=0\right.$ during this extra-time). Dashed lines represent the solution of case (a), depicted here for comparison.

On the other hand, case (c) shows that small variations of the switching times of the feedback control can produce huge qualitative differences in the behavior of the agents. We have introduced a small perturbation of the first interval of activation $S_{1}=[39.17,39.55]$, by keeping $\kappa=1$ until 39.6 instead of until 39.55 (a perturbation of relative size 1 $39.55 / 39.6=1.3 \times 10^{-3}$ ). For $t \geq 39.6$, we use again the feedback law (19), so that a different control function profile arises. The resulting behavior of the agents is depicted in Fig. 9(c).

Fig. 9(c) shows that after the perturbation, the agents describe a widely deviated trajectory, especially in the case of the driver, with respect to the one described with the unperturbed feedback law shown in Fig. 7. Accordingly, the perturbed control function profile, depicted in Fig. 8(c), is significantly different from the unperturbed feedback law. The driver requires more time to reach the target $\left(t_{f}^{\mathrm{c}}=t_{f}+1=64\right)$ than in cases (a) and (b). Compared with case (b), the cost is higher, although not excessively, $\mathcal{C}_{\mathrm{F}}^{(\mathrm{c})}=4.13$ (with no additional cost for the extra time because the evader moves in the right direction), and the number of ignition process is much smaller: $N_{\text {ig }}^{(\mathrm{c})}=16$.

Perturbations of the rest of switching times produce similar results, that is, qualitative deviations of the trajectories and a larger number of ignition processes, with respect to the unperturbed case (a), meaning that, in cases where delays can appear in the instants of time in which the control has to be manipulated, important changes of the behavior of the system may arise.

The oscillatory behavior arisen in case (c) can appear to be less convenient but, according to our numerical simulations, it is not necessarily worse than the smooth evaderfollowing trajectory; oscillations can contribute to reduce the number of ignition processes, with a low increase of the cost; see left panels (a) in Fig. 10.

The feedback control law is able to drive the evader along a given trajectory, provided 

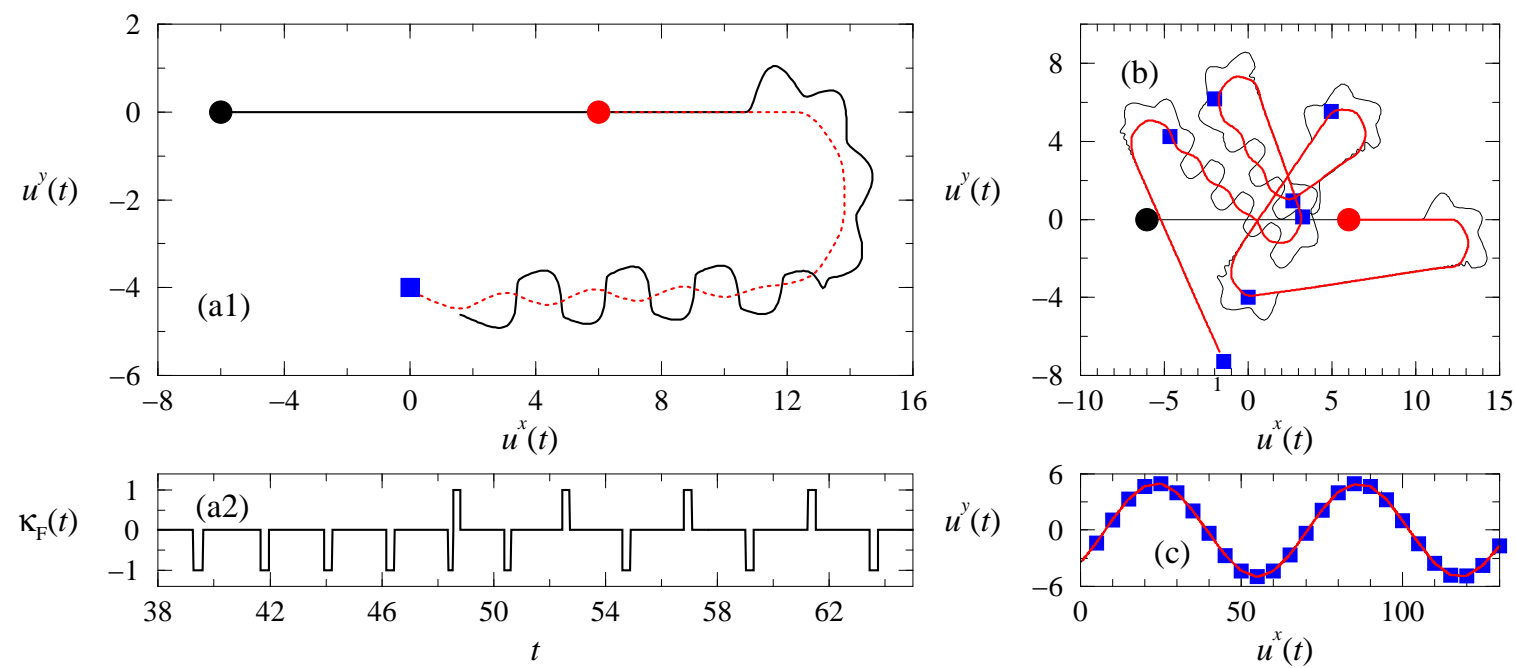

Figure 10: Left panels: (a1) agents' trajectories and (a2) feedback control producing an oscillatory behavior of the driver and a surprisingly low cost $\mathcal{C} \approx 4$.1. Panel (b): agent's trajectories in a case where the path to follow is a series of 7 targets randomly distributed in a radius smaller than 8 from the original location of the evader. Note that for some targets the trajectory is smooth $\left(T_{1}, T_{2}, T_{3}, T_{5}\right.$ and $\left.T_{7}\right)$ but for some others oscillations are necessary to adjust the alignment $\left(T_{4}\right.$ and $\left.T_{6}\right)$. Panel (c): evader's trajectory along a sinusoidal path described by a large number of closely spaced targets.

the trajectory is sufficiently smooth, that is, the trajectory can be described by a series of targets; see Fig. 10(b) for a series of random targets, and (c) for a sinusoidal trajectory described by a large number of closely spaced targets. The study of the behavior of the system when the path to follow has a very high curvature or describes very acute corners is matter for future work.

\section{Conclusion}

We have presented an agent based model for the guidance by repulsion problem, consisting of a system of equations corresponding to Newton's second law. The system can adopt two operating modes that can be controlled by a single parameter $\kappa(t)$ : for $\kappa=0$, the system is in the pure pursuit mode (control in mode OFF, i.e., resting state), and for $\kappa=1$, the system is in the circumvention mode (control in mode $\mathrm{ON}$, i.e., active state). We have shown that by appropriately defining the function $\kappa(t):\left[t_{0}, t_{f}\right] \rightarrow\{-1,0,1\}$, the driver can guide the evader to any desired target or along any (relatively smooth) path. We have then formulated an optimal control problem $(O C P)$ to find the optimal guidance strategy minimizing the cost in terms of the number of times the system is activated from its resting state and the time the system spends in the active mode. By means of (numerical) shooting methods, we have obtained the optimal open-loop strategies for the 
case where the number of activations is equal or smaller than one, finding that the system is highly sensitive to small variations of the activation/deactivation times.

These results show that open-loop controls would not be of practical interest in real problems due to the presence of external perturbations, thus suggesting the use of a feedback law. Taking advantage of the information provided in the study of the behavior of the system under open-loop controls for $N_{\text {ig }} \leq 1$, we have designed a feedback law for the case where $N_{\text {ig }}>1$, which allows to correct in real time for deviations from the desired trajectory. We have found that the feedback law is also highly sensitive to small variations of the conditions of the problem, in this case, of the accuracy with which the target is reached (i.e., the radius of the target ball). Moreover, our results show that the feedback law, and therefore the resulting behavior of the system, are highly sensitive to possible delays in the switching times of the control. This means that, in systems or devices where the manipulation of the control cannot be carried out at arbitrarily close instants of time, the behavior of the driver can exhibit large oscillations that can produce an increase of the cost. This may happen in situations where time delays exist in collecting and interpreting the data about the state of the system or in the reaction time of the system once the control is changed, especially when two consecutive changes are very close in time.

The main direction for the immediate future work consists in taking into account the cost for the driver to get close to the evader, both in time and travelled distance. This would correspond to add the cost of back propellers to the cost functional:

$$
J_{B}\left(t_{f}\right)=\eta_{1} \int_{t_{0}}^{t_{f}}\left\|\vec{u}_{d}(t)-\vec{u}_{d}\left(t_{0}\right)\right\|^{2} d t+\eta_{2} t_{f},
$$

and find a feedback control law for the two controls $\kappa(t)$ and $t_{f}$.

The interest of guidance by repulsion could also be extended to the case where the evader's behavior has a stochastic component and when multiple agents (evaders and/or drivers) are considered. The feedback law will be especially relevant when noise is considered in both the behavior of the agents and in the manipulation of the data.

\section{Acknowledgments}

We thank the anonymous reviewers for their interesting suggestions and for pointing out the interest of open problem which include the cost of back propellers in the cost functional. This material is based upon work that has received funding from the European Unions Horizon 2020 research and innovation programme under the Marie SkłodowskaCurie grant agreement No 655235, entitled "SmartMass", the Advanced Grant NUMERIWAVES/FP7-246775 of the European Research Council Executive Agency, the BERC 2014-2017 program and the pre-doctoral grant PRE-2014-1-461 of the Basque Government, the FA9550-15-1-0027 of AFOSR, the MTM2014-52347 and MTM2011-29306 Grants and the Severo Ochoa program SEV-2013-0323 of the MINECO, and a Humboldt Award at the University of Erlangen-Nürnberg. 


\section{A Asymptotic value of the velocities at long times when $\kappa=0$}

Numerical simulations show that when $\kappa(t)=0$ continuously for a sufficiently large time, both agent's velocities converge asymptotically to the same constant velocity $\vec{v}_{\text {sat }}$. In that state, $\dot{\vec{v}}_{d}(t)=\dot{\vec{v}}_{e}(t)=0$ and, from Eqs. (6) $-(7)$, we have

$$
-\frac{C_{D}^{E}}{\nu_{d}} \frac{\vec{u}_{d}-\vec{u}_{e}}{\delta_{\mathrm{as}}^{2}}\left[1+\frac{1}{\delta_{\mathrm{as}}^{2}}\left(\frac{C_{\mathrm{R}}}{C_{D}^{E}} \delta_{1}^{4}-\delta_{c}^{2}\right)\right]=-\frac{C_{E}^{D}}{\nu_{e}} \frac{\vec{u}_{d}-\vec{u}_{e}}{\delta_{\mathrm{as}}^{2}}=\vec{v}_{\mathrm{as}} .
$$

Thus, comparing norms, we obtain $v_{\mathrm{as}}=C_{E}^{D} /\left(\nu_{e} \delta_{\mathrm{as}}\right)$, and extracting $\delta_{\mathrm{as}}$, we obtain

$$
\delta_{\mathrm{as}}=\sqrt{\frac{\nu_{e}\left(C_{D}^{E} \delta_{c}^{2}-C_{\mathrm{R}} \delta_{1}^{4}\right)}{\nu_{e} C_{D}^{E}-\nu_{d} C_{E}^{D}}},
$$

provided $\nu_{e} C_{D}^{E}>\nu_{d} C_{E}^{D}$, as it is the case for the values we are considering. Note also that a necessary condition to have an effective short-range repulsion acting on the driver is that the factor between large parentheses in Eq. (6) is positive, so $C_{D}^{E} \delta_{c}^{2}-C_{\mathrm{R}} \delta_{1}^{4}>0$ and the radicand in expression (22) is positive.

\section{B On the controllability of the driver-evader system}

We show here that driver and evader are prevented from separating infinitely from each other and tend asymptotically to be separated a distance of order one. The proof follows the idea of "free agents" used in [24] (see also [27] for a more similar model).

Definition A driver agent $D$ is said to be a free agent at time $t$ if its distance to the evader $E$ is greater than an arbitrarily large positive constant $\delta \gg 1$; that is, $r(t)=$ $\left\|\vec{u}_{d}(t)-\vec{u}_{e}(t)\right\| \geq \delta$.

Proposition 1. If the driver $D$ is a free agent, then the system (11)-(4) can be reduced as follows:

$$
\begin{aligned}
& \dot{\vec{u}}_{d}(t)=\vec{v}_{d}(t) \\
& \dot{\vec{u}}_{e}(t)=\vec{v}_{e}(t) \\
& \dot{\vec{v}}_{d}(t)=-\frac{C_{D}^{E}}{m_{d}} \frac{\vec{u}_{d}(t)-\vec{u}_{e}(t)}{\left\|\vec{u}_{d}(t)-\vec{u}_{e}(t)\right\|^{2}}-\frac{\nu_{d}}{m_{d}} \vec{v}_{d}(t) \\
& \dot{\vec{v}}_{e}(t)=\frac{C_{E}^{D}}{m_{e}} \frac{\vec{u}_{e}(t)-\vec{u}_{d}(t)}{\left\|\vec{u}_{e}(t)-\vec{u}_{d}(t)\right\|^{2}}-\frac{\nu_{e}}{m_{e}} \vec{v}_{e}(t) .
\end{aligned}
$$

Proof. Using Remark 1. 
Lemma 2. If $m_{d} / \nu_{d}<m_{e} / \nu_{e}$, then, $\forall t>0$,

$$
\left\|\vec{v}_{e}(t)\right\|^{2} \leq \frac{C_{E}^{D}}{C_{D}^{E}} \frac{m_{d}}{m_{e}}\left\|\vec{v}_{d}(t)\right\|^{2} \Longrightarrow\left\|\vec{v}_{e}(t)\right\|^{2} \leq \frac{C_{E}^{D}}{C_{D}^{E}} \frac{\nu_{d}}{\nu_{e}}\left\|\vec{v}_{d}(t)\right\|^{2} .
$$

Definition Let $\mathbf{q}=\left(\vec{u}_{d}, \vec{v}_{d}, \vec{u}_{e}, \vec{v}_{e}\right) \in \mathbb{R}^{8}$ and $V(\mathbf{q}): \mathbb{R}^{8} \rightarrow \mathbb{R}$ be the following potential functional:

$$
V(\mathbf{q})=\ln \left(\left\|\vec{u}_{d}-\vec{u}_{e}\right\|\right)+\frac{1}{2} \frac{m_{d}}{C_{D}^{E}}\left\|\vec{v}_{d}\right\|^{2}-\frac{1}{2} \frac{m_{e}}{C_{E}^{D}}\left\|\vec{v}_{e}\right\|^{2} .
$$

Then, for free agents $($ i.e., $r(t) \geq \delta$ ) and under the hypotheses of Lemma 2, $V(\mathbf{q})$ is positive.

Theorem 3. If the driver $D$ is a free agent, then $V(\mathbf{q})$ is bounded from below and $\dot{V}(\mathbf{q})$ is negative along the agents' trajectories defined by the system (11)-(4). Consequently, $V(\mathbf{q}(t))$ converges in time to a minimum which is reached when the distance between both agents is $\delta$.

Proof. The time-derivative of $V(\mathbf{q})$ along the agents' trajectories is given by:

$$
\dot{V}(\mathbf{q})=\nabla_{\vec{u}_{d}} V \cdot \frac{d \vec{u}_{d}}{d t}+\nabla_{\vec{v}_{d}} V \cdot \frac{d \vec{v}_{d}}{d t}+\nabla_{\vec{u}_{e}} V \cdot \frac{d \vec{u}_{e}}{d t}+\nabla_{\vec{v}_{e}} V \cdot \frac{d \vec{v}_{e}}{d t},
$$

where $\quad \nabla_{\vec{u}_{d}} V=-\nabla_{\vec{u}_{e}} V=\frac{\vec{u}_{d}-\vec{u}_{e}}{\left\|\vec{u}_{d}-\vec{u}_{e}\right\|^{2}}, \quad \nabla_{\vec{v}_{d}} V=\frac{m_{d}}{C_{D}^{E}} \vec{v}_{d} \quad$ and $\quad \nabla_{\vec{v}_{e}} V=-\frac{m_{e}}{C_{E}^{D}} \vec{v}_{e}$.

Then:

$$
\begin{aligned}
\dot{V}(\mathbf{q})= & \frac{\vec{u}_{d}-\vec{u}_{e}}{\left\|\vec{u}_{d}-\vec{u}_{e}\right\|^{2}} \cdot \vec{v}_{d}+\frac{m_{d}}{C_{D}^{E}} \vec{v}_{d} \cdot\left(-\frac{C_{D}^{E}}{m_{d}} \frac{\vec{u}_{d}-\vec{u}_{e}}{\left\|\vec{u}_{d}-\vec{u}_{e}\right\|^{2}}-\frac{\nu_{d}}{m_{d}} \vec{v}_{d}\right) \\
& -\frac{\vec{u}_{d}-\vec{u}_{e}}{\left\|\vec{u}_{d}-\vec{u}_{e}\right\|^{2}} \cdot \vec{v}_{e}-\frac{m_{e}}{C_{E}^{D}} \vec{v}_{e} \cdot\left(-\frac{C_{E}^{D}}{m_{e}} \frac{\vec{u}_{d}-\vec{u}_{e}}{\left\|\vec{u}_{d}-\vec{u}_{e}\right\|^{2}}-\frac{\nu_{e}}{m_{e}} \vec{v}_{e}\right) \\
= & -\frac{\nu_{d}}{C_{D}^{E}}\left\|\vec{v}_{d}\right\|^{2}+\frac{\nu_{e}}{C_{E}^{D}}\left\|\vec{v}_{e}\right\|^{2},
\end{aligned}
$$

which, under the conditions of Lemma 2, is negative. Then $V(\mathbf{q})$ decreases and is bounded from below, so $V(\mathbf{q})$ has a minimum, which is reached when $\left\|\vec{v}_{d}(t)\right\|=\left\|\vec{v}_{e}(t)\right\|=0$ and $r(t)=\left\|\vec{u}_{d}(t)-\vec{u}_{e}(t)\right\|=\delta$ (which is the minimum value of $r(t)$ for a free agent).

Thus, agents are prevented from separating infinitely from each other because as soon as $r(t) \geq \delta$, the driver becomes a free agent and is forced to move back towards the evader, provided the balance between the mass and the friction of the agents verifies Lemma 2 , 


\section{Simple shooting method}

Once the evader is turning back towards the target, that is, $v_{e}^{x}(t)<0$, we check the direction of the velocity vector of the evader with the line $\overline{E T}$ described by the evader and the target. Then, if for some time $t \in\left(t_{0}, t_{f}\right)$ the vector $\vec{v}_{e}(t)$ points towards a point located below $T$, the tentative value of $t_{\text {OFF }}$ must be reduced; if instead, at the final time $t_{f}$, the vector $\vec{v}_{e}(t)$ points towards a point located above $T$, then the tentative value of $t_{\mathrm{OFF}}$ must be augmented.

That is: Given a value of $t_{\mathrm{ON}}$, take an initial value of $t_{\mathrm{OFF}}$ larger than $t_{\mathrm{ON}}$ and:

0 . Solve the system (1) -(5) with $\kappa_{t_{\mathrm{ON}}}^{t_{\mathrm{FF}}}(t)$ for $t \in\left(t_{0}, t_{f}\right)$ and anotate the value of $t_{b}$, which is the first time such that $v_{e}^{x}\left(t_{b}\right)<0$. If no such time is reached, this means that $t_{\mathrm{OFF}}<t_{b}$, so take a larger value of $t_{\mathrm{OFF}}$ and shoot again (goto 0 ).

1. For each time $t \in\left(t_{b}, t_{f}\right)$, evaluate the instantaneous alignment $\alpha(t)$ of the velocity vector of the evader $\vec{v}_{e}(t)$ with respect to the target point $\vec{u}_{T}$ :

$$
\alpha(t)=\left(u_{T}^{y}-u_{e}^{y}(t)\right) v_{e}^{x}(t)-\left(u_{T}^{x}-u_{e}^{x}(t)\right) v_{e}^{y}(t) .
$$

Then, if $\alpha(t)<0$, take a smaller value of $t_{\mathrm{OFF}}$ and shoot again (goto 0 ).

2. If at time $t_{f}$ the velocity vector of the evader is still pointing above the target, that is, $\alpha\left(t_{f}\right)>0$, then take a larger value of $t_{\mathrm{OFF}}$ and shoot again (goto 0 ).

The new value of $t_{\mathrm{OFF}}$ for the next shoot can be selected with a simple method (e.g., bisection).

Stop when $|\alpha(t)|<\epsilon$, for a small value of the tolerance $\epsilon$; the value of $t_{\mathrm{OFF}}^{*}\left(t_{\mathrm{ON}}\right)$ has been found, proceed to the next value of $t_{\mathrm{oN}}$.

\section{References}

[1] Tessier-Lavigne M, Goodman CS. The Molecular Biology of Axon Guidance. Science 15 (1996) $1123-33$.

[2] Rørth P. Whence Directionality: Guidance Mechanisms in Solitary and Collective Cell Migration. Developmental Cell 20 (2011) 9-18.

[3] Coppinger RP, Feinstein M. How dogs work. University of Chicago Press (2015). See also Coppinger RP, Coppinger L. Dogs: A Startling New Understanding of Canine Origin, Behavior and Evolution. Scribner, New York (2001).

[4] Senyei A, Widder K, Czerlinski G. Magnetic guidance of drug-carrying microspheres. J Appl Phys 49 (1978) 3578-83.

[5] Sharma S, Katiyar VK, Singh U. Mathematical modelling for trajectories of magnetic nanoparticles in a blood vessel under magnetic field. J Magnetism Magnetic Materials 379 (2015) 102-7.

[6] Bahat A, Caplan SR, Eisenbach M. Thermotaxis of Human Sperm Cells in Extraordinarily Shallow Temperature Gradients Over a Wide Range. PLoS ONE 7 (2012) e41915. 
[7] Moussaid M, Helbing D, Theraulaz G. How simple rules determine pedestrian behavior and crowd disasters. Proc Natl Acad Sci (PNAS) 108 (2011) 6884-8.

[8] Bellomo N, Dogbe C. On the modeling of traffic and crowds: A survey of models, speculations, and perspectives. SIAM Rev 53 (2011) 409-63.

[9] Moussaid M, Kämmer J, Analytis P, Neth H. Social Influence and the Collective Dynamics of Opinion Formation. PLoS ONE 8 (2013) e78433

[10] Garnier S, Combe M, Jost C, Theraulaz G. Do Ants Need to Estimate the Geometrical Properties of Trail Bifurcations to Find an Efficient Route? A Swarm Robotics Test Bed. PLoS Comput Biol 9 (2013) e1002903.

[11] Vicsek T, Zafeiris A. Collective motion. Physics Reports 517 (2012) 71-140.

[12] Helbing D. Traffic and Related Self-Driven Many-Particle Systems. Rev Mod Phys 73 (2001) 1067141.

[13] D'Orsogna MR, Chuang YL, Bertozzi AL, Chayes L. Self-propelled particles with soft-core interactions: patterns, stability, and collapse, Phys Rev Lett 96 (2006) 104302.

[14] Gueron S, Levin SA, Rubinstein DI. The dynamics of Herds: From individuals to Aggregations. J Theor Biol 182 (1996) 85-98.

[15] Levine H, Rappel W-J, Cohen I. Self-organization in systems of self-propelled particles. Phys Rev E 63 (2001) 017101.

[16] Mogilner A, Edelstein-Keshet L, Bent L, Spiros A. Mutual interactions, potentials, and individual distance in a social aggregation. J Math Biol 47 (2003 353-89.

[17] Perea L, Elosegui P, Gómez G. Extension of the Cucker-Smale Control Law to Space Flight Formations. J Guidance Control Dynam 32 (2009) 527-37.

[18] Lukeman R, Li YX, Edelstein-Keshet L. Inferring individual rules from collective behavior. Proc Natl Acad Sci (PNAS) 107 (2010) 12576-80.

[19] Sprott JC. Anti-Newtonian dynamics. Am J Phys 77 (2009) 783-7.

[20] Zhdankin V, Sprott JC. Simple predator-prey swarming model. Phys Rev E 85 (2010) 056209

[21] Chen H-Y, Leung K. Rotating states of self-propelling particles in two dimensions. Phys Rev E 73 (2006) 056107.

[22] Gazi V, Passino KM. Stability Analysis of Swarms. IEEE Trans on Automatic Control 48 (2003) $692-7$.

[23] Gazi V, Passino KM. A class of attraction/repulsion functions for stable swarm aggregations. Int J Control 77 (2004) 1567-79.

[24] Shi H, Xie G. Collective Dynamics of Swarms with a New Attraction/Repulsion Function. Math Probl Eng 735248 (2011) 021110.

[25] Liu B, Chu T-G, Wang L, Wang Z-F. Swarm Dynamics of a Group of Mobile Autonomous Agents. Chin Phys Lett 22 (2005) 254. 
[26] Gazi V, Passino KM. Swarm Stability and Optimization. Springer Science \& Business Media (2011).

[27] Escobedo R, Muro C, Spector L, Coppinger RP. Group size, individual role differentiation and effectiveness of cooperation in a homogeneous group of hunters. J Roy Soc Interface 11 (2014) 20140204.

[28] Shen J. CuckerSmale Flocking under Hierarchical Leadership. SIAM J Appl Math 68 (2007) 694719.

[29] Couzin ID, Krause J, Franks NR, Levin SA. Effective leadership and decision-making in animal groups on the move. Nature 433 (2005) 513-6.

[30] Aureli M, Porfiri M. Coordination of self-propelled particles through external leadership. EPL 92 (2010) 40004.

[31] Sontag ED. Mathematical control theory: deterministic finite dimensional systems. Springer Science \& Business Media (2013).

[32] Coron J-M. Control and Nonlinearity. Mathematical Surveys and Monographs 136, AMS (2007).

[33] Trélat E. Contrôle optimal : théorie \& applications. Vuibert, Collection "Mathématiques Concrètes" (2008) 2nd Ed.

[34] Lenhart S, Workman JT. Optimal Control Applied to Biological Models. Chapman and Hall/CRC (2007).

[35] Caponigro M, Fornasier M, Piccoli B, Trélat E. Sparse stabilization and optimal control of the Cucker-Smale model. Math Cont Related Fields 3 (2013) 447-66.

[36] Caponigro M, Fornasier M, Piccoli B, Trélat E. Sparse stabilization and control of alignment models. Math Mod Meth Appl Sci 25 (2015) 521-64.

[37] Borzi A, Wongkaew S. Modelling and control through leadership of a refined flocking system. Math Mod Meth Appl Sci 25 (2015) 255-82.

[38] Wang J, Li W. Motion patterns and phase-transition of a defender-intruder problem and optimal interception strategy of the defender. Comm Nonlinear Sci Numer Simulat 27 (2015) 294-301.

[39] Ghose K, Horiuchi TK, Krishnaprasad PS, Moss CF. Echolocating Bats Use a Nearly Time-Optimal Strategy to Intercept Prey. PLoS Biol 4 (2006) e108.

[40] Muro C, Escobedo R, Spector L, Coppinger RP. Wolf-pack (Canis lupus) hunting strategies emerge from simple rules in computational simulations. Behav Proc 88 (2011) 192-7.

[41] Isaacs R. Differential Games. John Wiley, New York (1965).

[42] Nahin PJ. Chases and Escapes: The Mathematics of Pursuit and Evasion. Princeton University Press (2007). 